\title{
Characterization of Sphere-like Structure in Aluminum based Alloy
}

\author{
Chunfei Li ${ }^{1}$, Limin Wang ${ }^{2}$, Helen Hampikian ${ }^{1}$, Matthew Bair ${ }^{1}$, Andrew Baker ${ }^{1}$, Andrew Sobolewski ${ }^{1}$, \\ Mingjian $\mathrm{Hua}^{3}$, Dingqiang $\mathrm{Li}^{4}$, and Qiongshu $\mathrm{Wang}^{3}$ \\ 1. Clarion University, Clarion, PA USA \\ 2. ChangChun Institute of Applied Chemistry, Chinese Academy of Science, Changchun, P.R. China \\ 3. University of Pittsuburgh, Pittsburgh, PA USA \\ 4. Youngstown State University, Youngstown, OH USA
}

$\mathrm{Al}_{65} \mathrm{Cu}_{25} \mathrm{Fe}_{15}$ is a well-known alloy composition for the preparation of stable icosahedral quasicrystalline phase (i-phase) [1]. It has been reported that the resulting phases could be complicated, depending on the precise composition and preparation process delicately. In this study, an alloy with nominal composition of $\mathrm{Al}_{65} \mathrm{Cu}_{25} \mathrm{Fe}_{15}$ was prepared by arc melting. Different from regular alloys, the ingot was found to be porous. A detailed morphology examination of the alloy surface by using Scanning Electron Microscope (SEM), sphere-like particles have been found. The main finding of the present study is that the surface of the sphere-like particles are decorated with multiple sets of concentric rings. The possible formation mechanisms of such rings are discussed.

The alloy was prepared by arc melting pure aluminum, copper, and iron. A TESCAN Vega-3 XMU SEM equipped with Oxford Aztec Energy Dispersive X-ray Spectroscopy (EDS) was used to examine the alloy surface morphology and carry out compositional analysis of individual particles. X-ray Diffraction (XRD) was performed on Bruker-Nonius D8 Advance Powder Diffractometer.

An SEM image of the alloy surface morphology is shown in Fig. 1(a). The arrow indicates one spherelike particle, which is the focus of the present study. The diameters of the sphere-like particles are in the order of $20 \mu \mathrm{m}$. Particles with distinct facets are considered as i-phase. A high resolution SEM image of such particle is shown in Fig. 1(b), several concentric ring sets were marked with arrows. A side-view SEM image of one ring set is shown in Fig. 1(c). The rings are terrace-like structure. The diameters of the most inner rings are in in the order of one micrometer while that of the most outer rings are in the order of five micrometers. The horizontal step size is approximately half a micrometer and the vertical step size is in the order of one hundred nanometers. Summarizing the results of EDS, X-ray diffraction, and Electron Back Scattering Diffraction, the crystalline structure of the sphere-like particles is considered as $\mathrm{AlFe}(\mathrm{Cu})$ phase $(\beta$-phase) of cubic structure [2].

The following reason is suggested for the formation mechanism of the sphere. As the alloy melt cools down, the solid i-phase with different composition from the alloy melt matrix precipitates first. In the present experimental condition, the i-phase particles separate from each other with residual liquid phase on the surfaces. Under the influence of surface tension, the liquid phase changes to spherical shape. As the temperature decreases further, the residual liquid phase transforms to cubic $\mathrm{AlFe}(\mathrm{Cu})$ phase. Two possible models are suggested for the formation of concentric ring sets. First, as the liquid sheet transforms to spherical shape, it causes oscillation which generates waves. Since the solidification of liquid drop happens quickly, these waves are frozen and later are observed as concentric ring sets. Second, the horizontal steps could correspond to the low energy plane of the $\mathrm{AlFe}(\mathrm{Cu})$ crystalline planes. The ring sets are formed to maximize the low surface energy planes and therefore, to lower the 
system energy. More studies need to be carried out to have a better understanding about the formation mechanism of the concentric ring sets. [3]

\section{References:}

[1] A.P. Tsai, Chem Soc Rev, 42 (2013) 5352.

[2] E. Huttunen-Saarivirta, J. Alloys Compd., 363 (2004) 150.

[3] The authors acknowledge funding from the National Science Foundation (DMR-1229063 AND DMR-1461607).
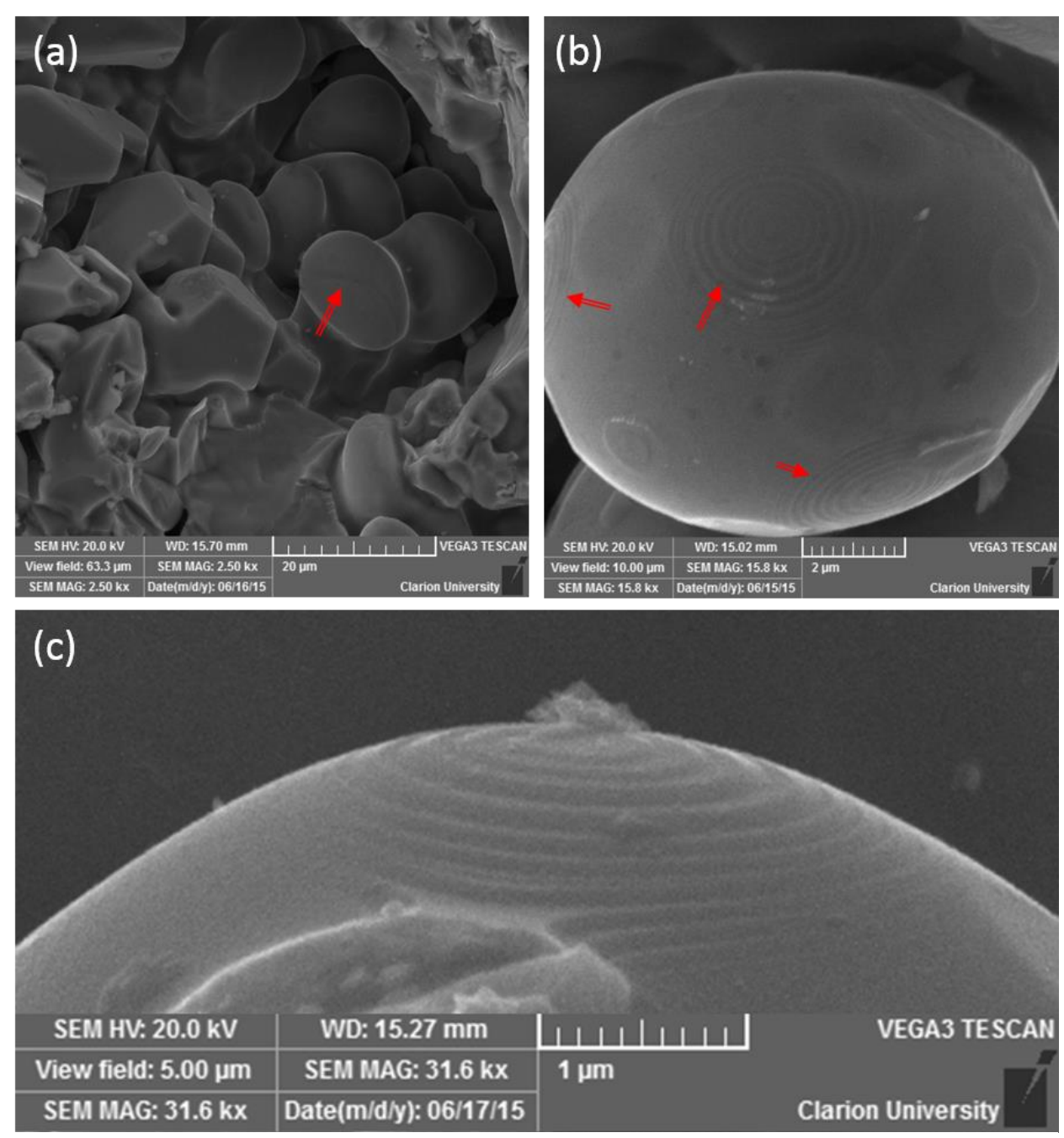

Figure 1. SEM images showing (a) the co-existence of sphere-like and i-phase particles, (b) concentric ring sets on the surface of sphere-like particle, and (c) side-view of a ring set. 